\title{
Outcome of mechanical heart valve replacement in children: A 20-Year Experience
}

\author{
Hong Ju Shin", Jo Won Jung, Han Ki Park, Young Hwan Park \\ From World Society of Cardiothoracic Surgeons 25th Anniversary Congress, Edinburgh \\ Edinburgh, UK. 19-22 September 2015
}

\section{Background/Introduction}

There are a few reports regarding outcome of mechanical valve replacement of children.

\section{Aims/Objectives}

We investigated our 20-year experience with pediatric mechanical heart valve replacement with respect to mortality, valve-related morbidity, and reoperation risk factors.

\section{Method}

The medical records of 42 patients (20 male, $47.6 \%$ ) who underwent 59 mechanical valve replacements between March 1992 and February 2014 were reviewed, retrospectively. Median age of the patients was 11.0 years (range, 9.1 months to 17.6 years) and 15 patients $(25.4 \%)$ were less than 5 years. Congenital valve disease was the most common diagnosis $(\mathrm{n}=16$, 27.6\%). Mitral valve replacement was performed in 39 patients, followed by aortic valve in 12 patients, pulmonic valve in five patients. Double valve replacements were performed in two patients. Mechanical valve was used in 51 cases $(83.6 \%)$. The median valve size was $23 \mathrm{~mm}$ (range, 16 to $33 \mathrm{~mm}$ ), and the median follow-up duration after valve replacement was 6.0 years (range, 15 days to 21.7 years). Events were defined as the following: thrombosis, embolism, bleeding, reoperation, and death.

\section{Results}

There were two in-hospital mortality (all low cardiac outputs) and two late deaths (sepsis after heart transplantation and heart failure at 5.4 and 14.0 years postoperatively, respectively). Survival rates were $96.6 \%$, $96.6 \%$, and $93.7 \%$, at 3,5 , and 10 years, respectively.

\footnotetext{
Severance Cardiovascular Hospital, 250 Seongsanno (134 Sinchon-dong),
} Seodaemun-gu, Seoul, Korea 